\title{
Characterization of two distinct, simultaneous hot electron beams in intense laser-solid interactions
}

\author{
B. I. Cho, ${ }^{1}$ J. Osterholz, ${ }^{1,2}$ A. C. Bernstein, ${ }^{1}$ G. M. Dyer, ${ }^{1}$ A. Karmakar, ${ }^{3}$ A. Pukhov ${ }^{3}$ and T. Ditmire ${ }^{1}$ \\ ${ }^{1}$ Texas Center for High Intensity Laser Science, Department of Physics, University of Texas, Austin, Texas 78712-0263, USA \\ ${ }^{2}$ Institute for Laser and Plasmaphysics, Heinrich-Heine-University, Geb. 25.33, Universitätsstr. 1, 40225 Düsseldorf, Germany \\ ${ }^{3}$ Institut für Theoretische Physik I, Heinrich-Heine-Universität, Universitätsstr. 1, 40225 Düsseldorf, Germany
}

(Received 13 May 2008; revised manuscript received 27 August 2009; published 11 November 2009)

\begin{abstract}
The transport of energetic electron beams generated from aluminum foils irradiated by ultraintense laser pulses has been studied by imaging coherent transition radiation from the rear side of the target. Two distinct beams of $\mathrm{MeV}$ electrons are emitted from the target rear side at the same time. This measurement indicates that two different mechanisms, namely resonance absorption and $\boldsymbol{j} \times \boldsymbol{B}$ heating, accelerate the electrons at the targets front side and drive them to different directions, with different temperatures. This interpretation is consistent with 3D-particle-in-cell simulations.
\end{abstract}

DOI: 10.1103/PhysRevE.80.055402

It is now well known that the irradiation of a solid target plasma with a high intensity laser leads to the production of beams of hot electrons [1-4], and these $\mathrm{MeV}$ electron beams have been often observed in various short pulse laser-plasma experiments with focal intensities above $10^{18} \mathrm{~W} / \mathrm{cm}^{2}$ [5-7]. Because of the generation of strong magnetic fields [3] and the behavior of return currents in the laser target material [8], the transport of these hot electrons is a complex subject and is still under investigation in many laboratories.

There are numerous ways hot electrons can be generated by an intense laser. These include resonance absorption, [9] in which the laser drives a plasma wave at the critical density surface in a plasma density gradient, and Brunel absorption (or vacuum heating), in which electrons are directly accelerated in the field of the laser at a sharp subwavelength-scale plasma-vacuum interface [10]. Hot electrons can also be driven ponderomotively by the $\boldsymbol{j} \times \boldsymbol{B}$ acceleration mechanism [1]. The former mechanisms will tend to accelerate electrons in a direction along a plasma density gradient (normal to the target surface), while the $\boldsymbol{j} \times \boldsymbol{B}$ mechanism will generate electrons in the direction of the laser's propagation. Santala et al. observed indirect evidence for these two mechanisms by measuring hard $\mathrm{x}$-ray generation from irradiation of solid targets [11] finding that a hard $\mathrm{x}$-ray beam was generated normal to the target surface when the plasma scale length was short $(\ll 10 \mu \mathrm{m})$, while $\mathrm{x}$-rays along the laser propagation direction were created when a longer scale-length $(>10 \mu \mathrm{m})$ preplasma was illuminated. Using Cerenkov radiation as a diagnostic [12] Ter-Avetisyan et al. observed two distinct beams of hot electrons traversing a transparent layer on the back side of their solid target [13].

A more direct way to measure the emergence of hot electrons from the back side of a target is by observing the transition radiation that is emitted when the hot electrons transit an interface in the dielectric function as they cross from the target material into vacuum. Santos et al. showed that by imaging this transition radiation, the electron spatial distribution emerging from the target can be accurately measured [14]. When the emerging hot electrons are bunched in a series of short pulses at multiples of the laser frequency, the emitted transition radiation can assume a narrow optical spectrum [15]. This coherent transition radiation (CTR) was
PACS number(s): 52.38. $-\mathrm{r}, 52.50 . \mathrm{Jm}$

observed by Zheng et al. at the laser wavelength which led them to conclude that the electron bunches were emitted at the laser frequency, a clear signature of resonance absorption [16]. Baton et al. and Popescu et al. observed CTR at the second and higher harmonics of the laser wavelength suggesting that this emission arose from hot electrons produced by $\boldsymbol{j} \times \boldsymbol{B}$ acceleration $[17,18]$.

In this Rapid Communication, we present measurements of CTR emission from the rear surface of aluminum foils irradiated by $40-\mathrm{fs}$ laser pulses at intensities up to 2 $\times 10^{19} \mathrm{~W} / \mathrm{cm}^{2}$. We observe two distinct lobes emerging simultaneously in the CTR emission pattern, which we attribute to resonance absorption and $\boldsymbol{j} \times \boldsymbol{B}$ heating. With this technique we have measured the relative efficiency of these two heating mechanisms, finding that about one order of magnitude more hot electrons were driven by resonance absorption than by $\boldsymbol{j} \times \boldsymbol{B}$ heating and that the hot electron temperatures of each population were $0.78 \pm 0.02 \mathrm{MeV}$ and $1.47 \pm 0.22 \mathrm{MeV}$ respectively. These results are compared with three-dimensional particle-in-cell (3D-PIC) simulations.

Our experimental data were acquired on the THOR laser at the University of Texas at Austin. This laser delivers pulses of $0.7 \mathrm{~J}$ with duration of $40 \mathrm{fs}$ at a wavelength 800 $\mathrm{nm}$. The $p$-polarized pulses were focused in the vacuum $\left(2 \times 10^{-5}\right.$ mbar) with an $f / 2.8$ off-axis parabolic mirror to a spot size of $7 \mu \mathrm{m}$ (full width at half maximum) onto flat aluminum foils with $45^{\circ}$ angle of incidence. Peak intensity was $2 \pm 0.5 \times 10^{19} \mathrm{~W} / \mathrm{cm}^{2}$. THOR has prepulses which arrive $20 \sim 50$ ps prior to the main pulse generating a preplasma of about $3 \mu \mathrm{m}$ (measured previously in the work of [19]). To measure CTR emission, the experimental setup described in [20] was employed. With a $20 \times$ achromat and $45^{\circ}$ beam splitter, the target rear side was imaged onto a charge coupled device (CCD) camera and the slit of a spectrometer simultaneously. Optical signals were filtered with bandpass filters to select specific CTR wavelengths.

A typical CTR image at $400 \mathrm{~nm}$ and spectrum of optical emission from the rear surface of $10-\mu$ m-thick aluminum foil are shown in Fig. 1. The narrow-band spectrum around $400 \mathrm{~nm}$, which is the half of the laser wavelength, confirms that this emission was CTR from microbunched electrons. Two bright spots (designated A and B in Fig. 1(a)) were 

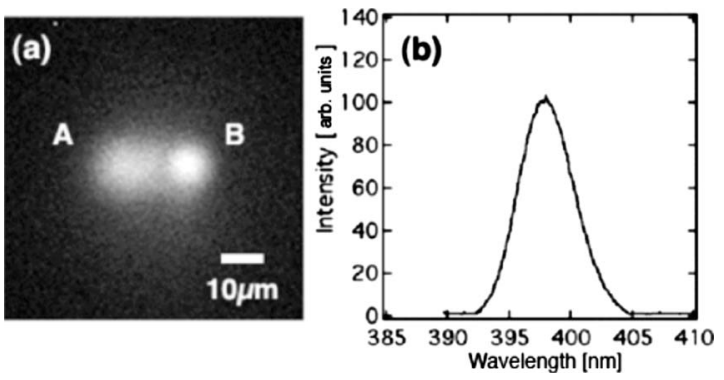

FIG. 1. Typical (a) image and (b) spectrum of $400 \mathrm{~nm}$ CTR optical emission from the rear side of a $10 \mu \mathrm{m}$ aluminum foil (tolerance of thickness $10 \%$ ). Two emissions in the images are denoted by spot $\mathrm{A}$, which is elliptical, and spot $\mathrm{B}$, which is circular. The separation of two spots in (a) is $12.6 \mu \mathrm{m}$.

observed at the same time, which we surmise to be the result of electron emitted in two different directions from a common source: the laser focal spot. Spot A arises from an electron beam propagating close to the target normal direction and spot $\mathrm{B}$ arises from an electron beam traveling in a direction close to the axis of laser. Emission from spot $\mathrm{A}$ is $25 \mu \mathrm{m}$ in diameter and light in spot B is $10 \mu \mathrm{m}$. We also observe strong CTR emission at $800 \mathrm{~nm}$, the fundamental wavelength of the laser. In this case only one spot is observed arising from an electron beam propagating normal to the target surface [21]. We attribute the emission from lobe A with electrons bunched at the laser frequency consistent with resonance absorption and emission in lobe B to arise from electrons bunched predominantly at twice the laser frequency indicating $\boldsymbol{j} \times \boldsymbol{B}$ acceleration. Measuring the emission size from different thickness of targets gives direct information on the divergences of the two electron beams. Electrons from resonance absorption exhibit a greater divergence $\left(\sim 6^{\circ}\right)$ than the $\boldsymbol{j} \times \boldsymbol{B}$ driven hot electrons $\left(\sim 4^{\circ}\right)$, which emerge from the back side with a size nearly the same as that of the laser focal spot. These small opening angles may be attributed to collimation of the electron beams by the self-generated magnetic fields [4]. Despite a longer traveling distance, the smaller divergence of the $\boldsymbol{j} \times \boldsymbol{B}$ case indicates that stronger magnetic fields and higher energy of electrons are produced ponderomotively than resonantly. The separation of the two spots is $11.7 \pm 3.1 \mu \mathrm{m}$, consistent with the difference in angle between normal and forward directed electrons passing through a $10-\mu$ m-thick target.

To characterize these two electron beams, we measured the fluence at the center of each CTR spot from 10- $\mu$ m-thick targets as a function of on-target laser intensity (changed by varying laser energy). The peak fluence of these spots is presented in the Fig. 2(a). We observed a sharp increase in CTR fluences from both lobes as laser intensity increased, with faster scaling in lobe B (the emission from electrons emitted along the laser axis). We fitted the scaling of CTR fluences in spots $\mathrm{A}$ and $\mathrm{B}$ to $\sim I^{\alpha}$, finding that $\alpha=4.6 \pm 0.3$ and $5.4 \pm 0.2$, for $\mathrm{A}$ and $\mathrm{B}$, respectively.

In order to understand the distinction in the two emission spots, CTR spectra were calculated using formulas developed by Zheng et al. [21]. The configuration for the calculation is depicted in the inset of Fig. 2(b). In the model, we consider hot electrons to originate from a point at the center
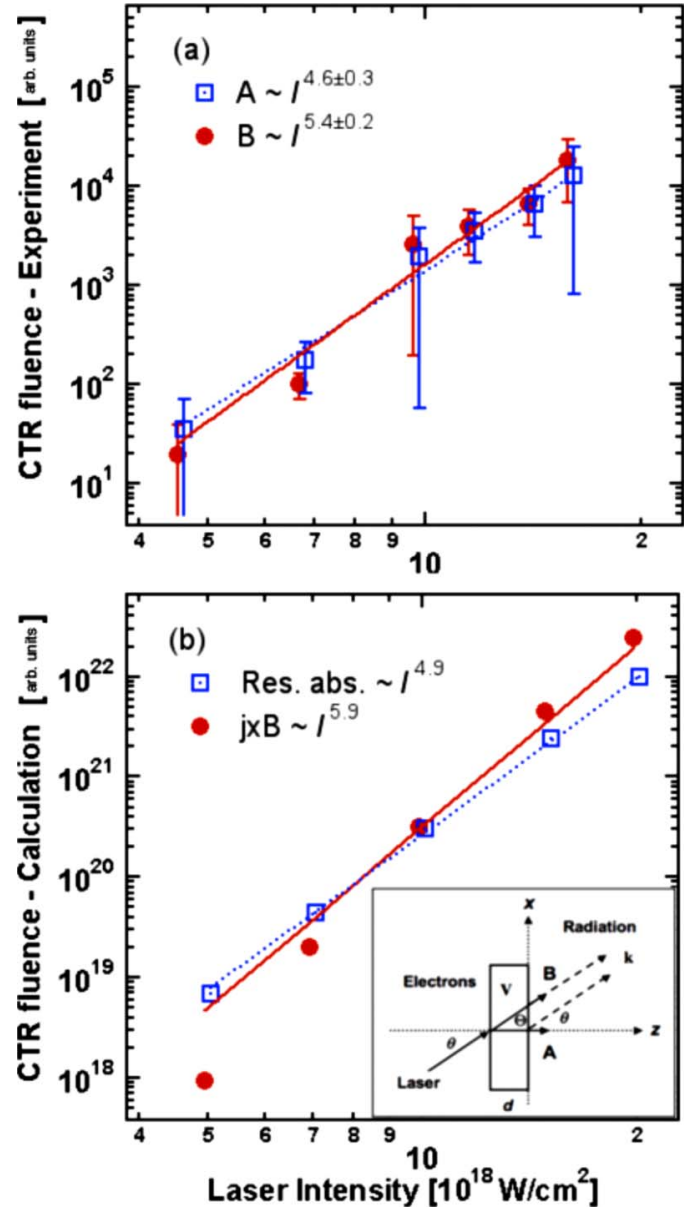

FIG. 2. (Color online) Dependence of CTR fluence around 400 $\mathrm{nm}$ on laser intensity: (a) experimental data and fitting lines and (b) theoretical calculations and fitting lines, where $10 \mu \mathrm{m}$ aluminum foil was irradiated with incidence angle of $45^{\circ}$. Each data point in (a) is an average of at least five shots and error bars represent the standard deviations. CTR fluences in (b) are calculated with several laser intensities and fitted. For clarity, data and fitting for A and resonance absorption have a slight offset of +0.1 in $x$ axis and data and fitting for $\mathrm{B}$ and $\boldsymbol{j} \times \boldsymbol{B}$ heating have an offset of -0.1 . (Inset) Configuration of the CTR calculation. Effective target thickness $d=13 \mu \mathrm{m}$, laser incidence angle and observation angle $\theta=45^{\circ}$.

of the laser focus on the target surface. We take the electrons to move ballistically on a line, which has an angle of $\Theta$ with respect to the $z$ axis. The CTR spectrum $\varepsilon_{\text {CTR }}$ is then given by

$$
\begin{aligned}
\frac{d^{2} \varepsilon_{\mathrm{CTR}}}{d \omega d \Omega} \sim & N_{b}^{2} \mid \int d \tau d \beta e^{i \omega \tau} \sum_{n=1}^{\Lambda} \delta\left(\tau-\tau_{n}-t_{0} / \beta\right) \\
& \times\left.\frac{\beta \cos \Theta(\sin \theta-\beta \sin \Theta) f_{\mathbf{v}}(\beta)}{\left[(1-\beta \sin \theta \sin \Theta)^{2}-\beta^{2} \cos ^{2} \theta \cos ^{2} \Theta\right]}\right|^{2},
\end{aligned}
$$

where $N_{b}$ is the electron number in each electron bunch, $\Lambda$ is a total number of electron bunches, $\theta$ is angle of observation, $t_{0}$ is the time when the fastest electron reaches the target rear side, $\tau_{n}$ is the time when the $n$th electron bunch is generated, 
$\beta$ is speed of electrons in the units of $c$, and $f_{\mathbf{v}}(\beta)$ is the electron velocity distribution. We assume that the hot electrons in each electron bunch have a relativistic Boltzmann velocity distribution with temperature $T$.

The scalings of the CTR emission at the second harmonic frequency of the laser with laser intensity $I$ have been calculated for the two lobes and are presented in Fig. 2(b). To calculate the CTR fluence scaling of resonance absorption, each electron bunch is spaced one per laser cycle and is assumed to have a temperature given by the empirical formula of Beg et al. [22] $T_{\text {res }}=0.1\left(I_{17} \lambda_{\mu m}^{2}\right)^{1 / 3} \mathrm{MeV}$, where $I_{17}$ is the laser intensity in units of $10^{17} \mathrm{~W} / \mathrm{cm}^{2}$ and $\lambda_{\mu m}$ is the laser wavelength in $\mu \mathrm{m}$. $\Lambda$ is set to 15 for the $40 \mathrm{fs}$ laser pulse employed in the experiment. Using this theory, we calculated the CTR spectrum and find, consistent with our data, that it is dominant at the laser frequency $\omega_{0}$ but also has significant radiation at $2 \omega_{0}$. To estimate the electron temperature arising from $\boldsymbol{j} \times \boldsymbol{B}$ heating, where two electron bunches per laser cycle are injected along the laser propagating direction $\left(\Theta=45^{\circ}\right)$, we use the formula of Wilks [1] $T_{p m}=0.511\left(\sqrt{1+I_{17} \lambda_{\mu \mathrm{m}}^{2} / 11.3}-1\right) \mathrm{MeV}$ and set the number of bunches $\Lambda$ to 30 (twice the number of optical cycles in our pulse). In this case, there is no significant radiation at $\omega_{0}$ (also consistent with our experimental observation). Calculated values were fitted to the scaling $I^{\alpha}$ and yield exponential indices of 4.9 for resonance absorption and 5.9 for $\boldsymbol{j} \times \boldsymbol{B}$ heating, remarkably close to those found experimentally for the two lobes, lending strong evidence that lobe $\mathrm{A}$ arises from resonance absorption and lobe $\mathrm{B}$ arises from $\boldsymbol{j} \times \boldsymbol{B}$ heating.

When the electron number $N_{b}$ is set the same for both cases, the calculated $400 \mathrm{~nm}$ CTR fluence for the resonance absorption lobe is about two orders of magnitudes lower than from the $\boldsymbol{j} \times \boldsymbol{B}$ emission lobe. As shown in the experimental data, the two CTR signals have the same strength at a laser intensity of $\sim 8 \times 10^{18} \mathrm{~W} / \mathrm{cm}^{2}$. Using Eq. (1), we derived the ratio of the electron numbers in a single bunch generated by resonance absorption $N_{b, R A}$ and $\boldsymbol{j} \times \boldsymbol{B}$ heating $N_{b, j \times B}$. The

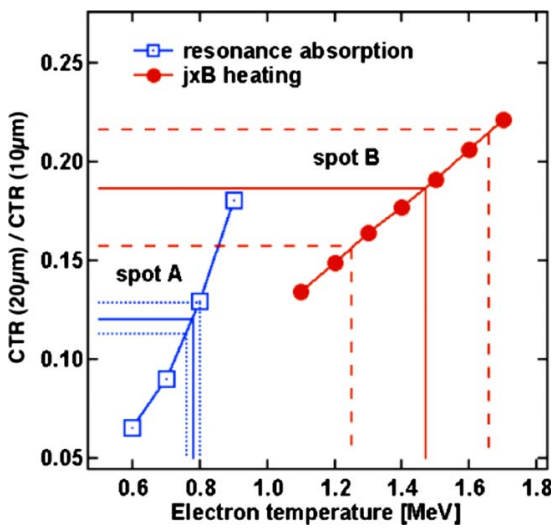

FIG. 3. (Color online) $2 \omega_{0}$ CTR ratios from different thickness of targets, 10 and $20 \mu \mathrm{m}$. Squares and rounds are calculated values for resonance absorption and $\boldsymbol{j} \times \boldsymbol{B}$ heating. The solid horizontal lines depict measured ratios for spots $A$ and $B$ in the experiment and the verticals represent corresponding electron temperatures. Dotted lines are the errors generated from twelve $10 \mu \mathrm{m}$ shots and ten $20 \mu \mathrm{m}$ shots. best agreement between the calculated and measured CTR fluences in the range of laser intensities shown in Fig. 2 was obtained for $N_{b, R A}=10.6 \times N_{b, j \times B}$, indicating that our laser interaction drives about one order of magnitude more hot electrons by the resonance absorption mechanism than by $\boldsymbol{j}$ $\times \boldsymbol{B}$ acceleration.

In order to determine the temperature of each electron beam experimentally, the CTR fluences of the two spots from both 10 and $20 \mu \mathrm{m}$ (effectively 13 and $23 \mu \mathrm{m}$, including the $3 \mu \mathrm{m}$ plasma length) thick aluminum foils were measured and compared. The CTR strength will decrease as electrons propagate further since velocity dispersion of the finite temperature bunches causes a spread of each bunch. There-
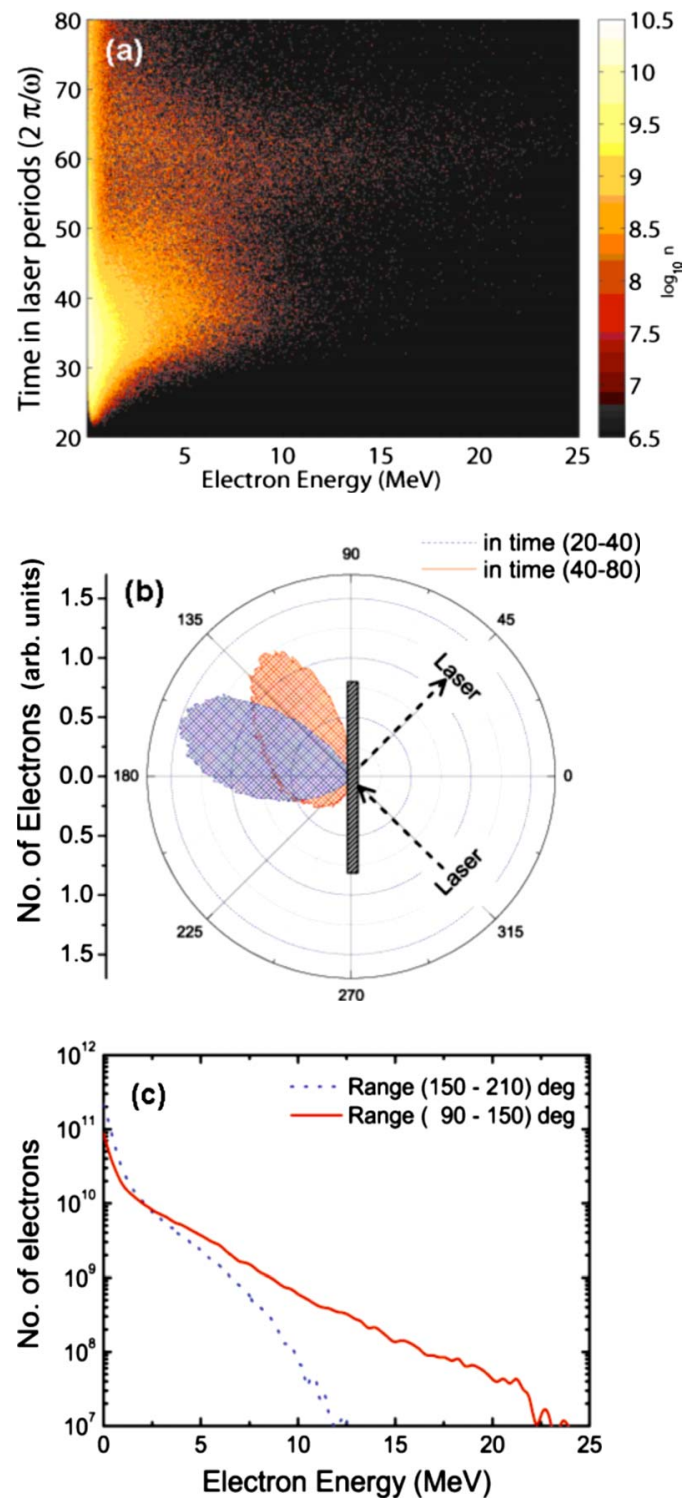

FIG. 4. (Color online) (a) Energy phase spectrum of the hot electrons coming out of the target rear surface. The color bar denotes the logarithm of density of the hot electrons. (b) Angular divergence of the hot electrons of the two different time regimes of (a). (c) Energy spectrum of the two streams of electrons as shown in (b). The scale around the circumference denotes the angle of emission and the scale on the left shows the radial scale of electrons emitted per unit angle. 
fore a drop in CTR fluence with increasing target thickness can be used to determine the hot electron temperature [20]. For spots A and B at a laser intensity of $2 \times 10^{19} \mathrm{~W} / \mathrm{cm}^{2}$, CTR fluences from $20 \mu \mathrm{m}$ targets were 0.120 and 0.186 times that from $10 \mu \mathrm{m}$ targets, respectively. Predicted CTR ratios $(20 \mu \mathrm{m} / 10 \mu \mathrm{m})$ as a function of temperature for the two electron beams were calculated using Eq. (1) and are plotted in Fig. 3. These calculations are compared with our measured ratios, which are depicted by the horizontal lines in this figure. This analysis indicates that the electron beam generated by resonance absorption had a temperature of $0.78 \pm 0.02 \mathrm{MeV}$, while the $\boldsymbol{j} \times \boldsymbol{B}$ beam exhibited a higher temperature of $1.47 \pm 0.22 \mathrm{MeV}$. At a laser intensity of 2 $\times 10^{19} \mathrm{~W} / \mathrm{cm}^{2}$, electron temperatures predicted by Eq. (1) are $0.5 \mathrm{MeV}$ for resonance absorption and $1 \mathrm{MeV}$ for $\boldsymbol{j} \times \boldsymbol{B}$ heating, consistent with our experiment.

As a final confirmation of the interpretation of our data, we have performed 3D-PIC simulations of hot electron generation from solid target plasmas with our experimental conditions using the parallelized relativistic code virtual laser plasma laboratory (VLPL) [23]. In this simulation, $p$-polarized laser pulses with a wavelength of $800 \mathrm{~nm}$ and a peak intensity of $5.4 \times 10^{19} \mathrm{~W} / \mathrm{cm}^{2}$ were obliquely incident at $45^{\circ}$ on an overdense plasma layer of $9 \mu \mathrm{m}$ thickness. The target density was ramped from 0 to 20 times critical density over $3.5 \mu \mathrm{m}$ (simulating our preplasma). The laser pulse was simulated as Gaussian in space and time with a duration of $30 \mathrm{fs}$ and spot radius of $6.7 \mu \mathrm{m}$. The entire simulation box was sampled with a grid of $250 \times 237 \times 20$ cells with eight particles per cell.

The temporal variation in the electron energy spectrum emerging from the target back surface is plotted in Fig. 4(a). This shows hot electrons emerging at two different times (roughly separated by 40 laser periods). Hot electrons emerging at the earlier time are greater in number but have lower maximum energies than the electrons coming later (attributed to resonance absorption). The angular divergence of these two streams of electrons, illustrated in Fig. 4(b), shows that these two bunches emerge in two distinct angular cones, one along the target normal on the rear surface and the other along the laser propagation direction (though at two different times). This distribution is remarkably similar to the experimental findings of Fig. 1. (It is interesting to note that the earlier electron population is emitted at a central angle which deviates somewhat from the normal. This may simply result from the fact that the early time bin used to distinguish these electrons may also include some $\boldsymbol{j} \times \boldsymbol{B}$ electrons emitted in the laser direction, distorting the angular distribution in that direction.)

In Fig. 4(c) we have plotted the energy spectrum in each electron emission cone, integrated over the time of the full simulation. The electrons following the laser propagation direction have an electron temperature of $3.3 \mathrm{MeV}$, which is about twice as high as the temperature of electrons emitted normal to the target surface (where $T_{\text {hot }}=1.4 \mathrm{MeV}$ ) and are about four times as numerous as the normal direction electrons. These trends are in good agreement with the experimental findings. The reason for the temporal difference between the two hot electron production mechanisms is not clear and requires further simulations. It may result from ponderomotive steepening of the plasma such that resonance absorption occurs earlier in the pulse before much steepening has occurred while the $\boldsymbol{j} \times \boldsymbol{B}$ heating predominates once the scale length has been steepened.

In summary, we have observed optical CTR from the target rear surface of aluminum foils irradiated with ultraintense laser pulses. We observe two distinct lobes in the CTR emission spots with narrow-band spectrum at the half wavelength of the laser. The analysis indicates that the two emission lobes are correlated with resonance absorption and $\boldsymbol{j} \times \boldsymbol{B}$ heating. We find that resonance absorption dominates the hot electron production by about an order of magnitude although with a temperature which is about half that of the electrons in the $\boldsymbol{j} \times \boldsymbol{B}$ peak. These findings appear to be completely consistent with three-dimensional PIC simulations of the experiment.

This work was supported by the DOE Office of Fusion Energy Science under a Fusion Science Center and the National Nuclear Security Administration under Cooperative Agreement No. DE-FC52-03NA00156.
[1] S. C. Wilks et al., Phys. Rev. Lett. 69, 1383 (1992).

[2] W. L. Kruer and K. Estabrook, Phys. Fluids 28, 430 (1985).

[3] A. Pukhov and J. Meyer-ter-Vehn, Phys. Plasmas 5, 1880 (1998).

[4] M. Tatarakis et al., Phys. Rev. Lett. 81, 999 (1998).

[5] G. Malka, E. Lefebvre, and J. L. Miquel, Phys. Rev. Lett. 78, 3314 (1997).

[6] T. Feurer et al., Phys. Rev. E 56, 4608 (1997).

[7] K. B. Wharton et al., Phys. Rev. Lett. 81, 822 (1998).

[8] M. Sherlock et al., Phys. Plasmas 14, 102708 (2007).

[9] W. L. Kruer, The Physics of Laser Plasma Interactions (Westview Press, Boulder, CO, 2003).

[10] F. Brunel, Phys. Rev. Lett. 59, 52 (1987).

[11] M. I. K. Santala et al., Phys. Rev. Lett. 84, 1459 (2000).
[12] F. Brandl et al., Europhys. Lett. 61, 632 (2003); J. Stein et al., Laser Part. Beams 22, 315 (2004).

[13] S. Ter-Avetisyan et al., Phys. Rev. E 77, 016403 (2008).

[14] J. J. Santos et al., Phys. Rev. Lett. 89, 025001 (2002).

[15] J. J. Santos et al., Phys. Plasmas 14, 103107 (2007).

[16] J. Zheng et al., Phys. Rev. Lett. 92, 165001 (2004).

[17] S. D. Baton et al., Phys. Rev. Lett. 91, 105001 (2003).

[18] H. Popescu et al., Phys. Plasmas 12, 063106 (2005).

[19] H. A. Sumeruk et al., Phys. Plasmas 14, 062704 (2007).

[20] B. I. Cho et al., J. Opt. Soc. Am. B 25, B50 (2008).

[21] J. Zheng et al., Phys. Plasmas 10, 2994 (2003).

[22] F. N. Beg et al., Phys. Plasmas 4, 447 (1997).

[23] A. Pukhov, J. Plasma Phys. 61, 425 (1999). 